\title{
Clinical evaluation of extracellular amino acids in severe head trauma by intracerebral in vivo microdialysis
}

\author{
R Kanthan, A Shuaib
}

\begin{abstract}
The underlying mechanisms of neuronal dysfunction in head injury are multifactorial and not fully understood. Recent experimental evidence indicates that excitatory amino acids-for example, glutamate-may play a key part in secondary neuronal damage after head injury. A patient with severe head trauma was monitored for extracellular amino acids by intracerebral in vivo microdialysis. Very high concentrations of glutamate, glycine, and $\gamma$-aminobutyric acid were recorded in the microdialysate specimens collected over three hours. In vivo microdialysis in head injury may prove to be a useful tool in understanding the pathogenesis of cell death in head trauma.
\end{abstract}

(F Neurol Neurosurg Psychiatry 1995;59:326-327)

Keywords: head injury; glutamate; in vivo microdialysis

Microdialysis is a relatively new technique which enables us to study the neurochemistry in living, freely mobile animals. This technique has been used in three patients with severe head trauma, in whom in vivo microdialysis was performed and energy related metabolites (lactate:pyruvate ratio and hypoxanthine concentrations) in the brain were studied. ${ }^{1}$ We wish to report a patient with severe head injury in whom glutamate, glycine, and $\gamma$-aminobutyric acid (GABA) were measured by in vivo microdialysis for four hours before his death.

Saskatchewan Stroke Research Centre and Departments of Medicine (Neurology), College of Medicine, University of Saskatchewan, Saskatoon, Saskatchewan, Canada

R Kanthan

A Shuaib

Correspondence to: Dr Ashfaq Shuaib, Saskatchewan Stroke Research Centre, Saskatoon, Saskatchewan, Canada.

Received 3 October 1994 and in final revised form 13 March 1995 Accepted 16 May 1995

\section{Case report}

A nineteen year old boy was admitted to the intensive care unit after a severe motor vehicle accident. Examination showed gross facial wounds and signs of severe diffuse closed head injury. He had absent pupillary reflexes and positive Doll's sign, and his responses to painful stimuli were minimal. He was kept in the intensive care unit for 24 hours at the end of which his organs were harvested. Intracerebral microdialysis was carried out for four hours through a Codman bolt inserted in the right frontal lobe. This initially provided access for intracranial pressure monitoring which averaged $20-25 \mathrm{~mm} \mathrm{Hg}$. He fulfilled the brain stem death criteria two hours after completion of the microdialysis. The microdialysates recorded extremely high concentrations of glutamate throughout the four hours of sampling, averaging over $3000 \mathrm{pMol} / 10 \mu \mathrm{l}$. This compares with normal values of 20 to 30 $\mathrm{pMol} / 10 \mu \mathrm{l}^{2}$

The use of microdialysis in humans is approved by the University advisory committee on ethics in human experimentation. Informed consent was obtained from the patient's parents before the study.

\section{Methods}

\section{Microdialysis}

We used custom designed CMA 10 (Stockholm, Sweden) probes for all the analyses. Sterile Ringer's solution was infused at a rate of $2 \mu \mathrm{l} / \mathrm{min}$ through a CMA-120 (Stockholm, Sweden) pump. The fluid was collected on ice and immediately transferred for analysis by high performance liquid chromatography (HPLC). Collections for the first hour after probe insertion were discarded to exclude erroneously high concentrations of neurotransmitters due to implantation trauma. The fluid was collected in 10 minute fractions.

\section{HPLC Analysis}

The microdialysis fluid was analysed for amino acids by HPLC and electrochemical detection. Measurements were by the methods of Donzani and Yamamoto ${ }^{3}$ with $o$-pthalaldehyde 2-mercaptoethanol before detection with a 715 Ultra Wisp sample processor (Waters, Toronto, Canada). A baseline Workstation data processing system (Waters, Toronto, Canada) was used for data storage 
Dialysate concentrations of glutamate, glycine, and $G A B A$ in head trauma as measured by intracerebral in vivo microdialysis

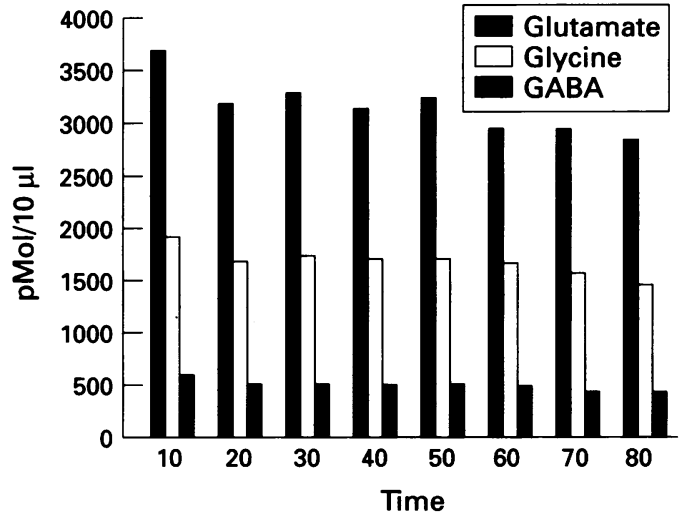

The concentrations of extracellular GABA were in the range $300-500 \mathrm{pMol} /$ $10 \mu \mathrm{l}$. The glutamate:GABA ratio remained high in all the dialysate samples.

\section{Discussion}

The role of glutamate leading to excitotoxicity and cell death in cerebral ischaemia is well established. ${ }^{4}$ The increased glutamate results from depolarisation of membranes (secondary to decreased availability of high energy compounds such as ATP), and a decreased reuptake mechanism mediates its toxicity by activating the postsynaptic receptors resulting in cellular oedema and accumulation of intracellular calcium leading to cell death.

In the fluid percussion model of experimental head trauma, MK-801, a noncompetitive N-methyl-D-aspartate (NMDA) antagonist attenuates the development of focal brain oedema. ${ }^{5}$ These data provide the clinical basis for the use of NMDA receptor antagonists in stroke and head trauma. Our evidence suggests that increased glutamate may be one of the major pathways leading to neuronal death in head injury. The increase in glutamate in head injury may be a primary response to the initial head trauma or it may be secondary to a "hypoxic ischaemic" state resulting from brain oedema or vasospasm response to the primary head injury insult.

This increase in glutamate has also been documented after concussive brain injury. It is well recognised that the extracellular space shrinks after such injury, resulting in a relative increase in the concentration of substances. In addition, changes in the capillary permeability after injury results in a relative increase in the concentrations of amino acids in the extracellular space. Nevertheless, these two mechanisms do not seem to be the major cause of the changes in the extracellular glutamate in brain injury. ${ }^{6}$

In vitro studies of neuronal cell cultures clearly show the toxicity of glutamate at concentrations of $50-100 \mu \mathrm{M} .^{7}$ Although, glutamate is the presumed mediator, other neurotransmitters may modulate its effect. In particular, GABA may counteract the glutamate induced excitation. ${ }^{2}$ In our case the very high glutamate: GABA ratio leads us to believe that this is a glutamate induced GABA release. It is possible that altered capillary permeability or an impaired blood brain barrier may have contributed to the very high concentrations recorded in our case.

In conclusion, we believe that intracerebral in vivo microdialysis may be a useful tool in understanding the pathogenesis of neuronal response in patients with head injury.

1 Persson L, Hillered L. Chemical monitoring of neurosurgical intensive care patients using intracerebral surgical intensive care patients using
microdialysis. $\mathcal{F}$ Neurosurg 1992;76:72-80.

2 During MJ, Spencer DD. Extracellular hippocampal glutamate and spontaneous seizure in the conscious glutamate and spontaneous seizure in
human brain. Lancet 1993;341:1607-10.

3 Donzanti BA, Yamamoto BK. An improved and rapid HPLC-EC method for the isocratic separation of amino acid neurotransmitters from brain tissue and microdialysis perfusates. Life Sci 1988;43:913-22.

4 Choi DW, Rothman SM. The role of glutamate neurotoxicity in hypoxic-ischemic neuronal death. Ann Rev Neurosci 1990;13:171-82.

5 Faden AL, Demediuk P, Panter SS, Vink R. The role of excitatory amino-acids and NMDA receptors in traumatic brain injury. Science 1989;244:798-800.

6 Katayama Y, Becker DP, Tamura T. Massive increase in extracellular potassium and indiscriminate release of glutamate following concussive injury. $f$ Neurosurg glutamate following

7 Choi DW, Maulucci-Gedde M, Kriegstein AR. Glutamate neurotoxicity in cortical cell culture. $\mathcal{f}$ Neurosci 1987; 7:357-68. 